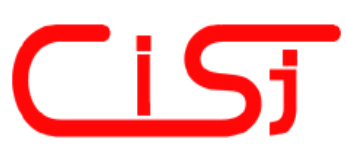

\title{
DAMAGE ANALYSIS OF CONCRETE STRUCTURES BY MEANS OF B-VALUE TECHNIQUE
}

\author{
Domenico Luca Carnì ${ }^{1)}$, Carmelo Scuro ${ }^{2)}$, Francesco Lamonaca ${ }^{3)}$, \\ Renato S. Olivito ${ }^{2)}$, Domenico Grimaldi ${ }^{1)}$

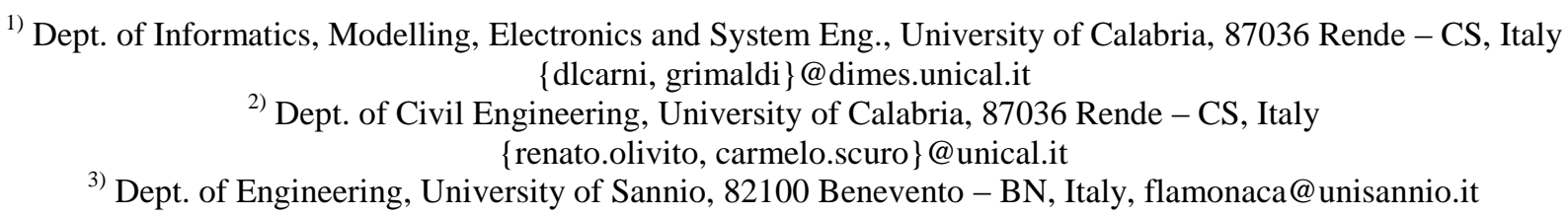

\begin{abstract}
The paper deals with the non-destructive monitoring of civil engineering structures damage by the Acoustic Emission (AE) of the cracks that can occur inside the concrete material. The damage of the concrete material has been evaluated by analyzing $\mathrm{AE}$ signals during the compression tests. In order to prevent the loss or partial acquisition of signals a multi-triggering acquisition modality and adaptive evaluation of the Acquisition Time Interval (ATI) have been used.

The analysis is based on the Gutenberg-Richter law (GBR), that is the relationship between the magnitude and total number of events of earthquakes in established region and time period. Through the GBR law the $b$-value was valued and used to select the critical AE signals that identify damage events.

Experimental tests have allowed to highlight correspondence between the critical AE signals and the damage in the material. Copyright $(\subset)$ Research Institute for Intelligent Computer Systems, 2017. All rights reserved.
\end{abstract}

Keywords: Acoustic Emissions, Compression Tests, Concrete, Gutenberg-Richter law, B-Value, Damage.

\section{INTRODUCTION}

Damage evaluation by Acoustic Emission (AE) signals is a promising technique for the noninvasive and continuous monitoring of concrete structures [1-3]. The AEs are used in order to realize noninvasive and continuous structural health monitoring system [4-11], and to obtain the state of stress in different types of materials [12-19].

The AE refers to the generation of elastic transient waves produced by a sudden distribution of stress in a raw material. When the materials are subjected to an external stress, as pressure, load, or temperature, the creation of damages inside the material can occur. The creation of damages acts as localized sources that trigger the release of energy, in the form of stress waves, which propagate to the surface. Such waves can be recorded by piezoelectric sensors.

The spread of the acoustic waves depends on the material and its nature. For example, the propagation velocity is greater in a homogenous material and it is reduced in discontinuous materials, such as concrete. The failure mode that occurs in the brittle materials, as concrete and bricks, is unpredictable and the AEs signals are subject to attenuation due to propagation and alteration [3]. This can provoke that $\mathrm{AE}$ signal reaches the transducer with low amplitude and the system does not identify the signal because it is corrupted by noise. Moreover, if the signal amplitude is below the trigger level the signal loss occurs, while the occurrence of multiple $\mathrm{AE}$ events can cause the loss or partial acquisition of signal due to the unpredictability of the Acquisition Time Interval (ATI). In order to reduce the possibility of signals loss, more piezoelectric transducers can be used. Moreover, the multi-triggering acquisition modality and adaptive evaluation of the ATI have been used, with the proper hardware pointed out: Logic Flat Amplifier and Trigger (L-FAT) generator block [1,2].

Fig. 1 shows an actual $\mathrm{AE}$ signal in which specific characteristic parameters are highlighted such as the maximum amplitude (Adm), the threshold noise for the identification of the $\mathrm{AE}$ event, and the discrimination between the hits and the noise. These parameters are used to characterize the fracture generating the AE signals and then to assess the damage in concrete specimen.

The $\mathrm{AE}$ are similar to the $\mathrm{P}$ waves that are generated during the earthquake. 
According to this similarity, in the paper, the damage of the concrete material has been evaluated by analyzing $\mathrm{AE}$ signals during the compression tests on the basis of the Gutenberg-Richter (GBR) law [21], that is the relationship between the magnitude and total number of events of earthquakes in established region and time period.

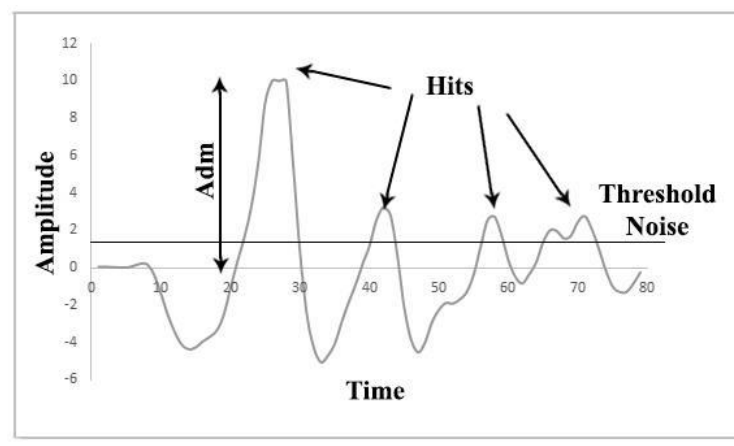

Fig. 1 - Characteristic parameters of AE signal

Through the GBR law the b-value was valued and used to select the $\mathrm{AE}$ signals that identify critical damage events.

The paper is organized as it follows. In Section 2, the GBR law is analyzed. In Section 3, information about the experimental setup and the correspondence between the critical AE signals selected on the basis of GBR law and specimen stress is synthetized. Finally, in Section 4 the conclusions are drawn.

\section{GBR LAW}

The study of the distribution of earthquake magnitude in size and time in a specifically region is analyzed usually though two statistical methods. The first one is based on the theory of extreme values and uses the maximum value of the magnitude [20]. The second one, taken into account in this paper, is based on the GBR law. It is based on the analysis of magnitude-frequency relationship and uses all magnitude values of earthquakes in a region and not just the highest ones [21].

The GBR law was used typically in seismology for analyzing the relationship between the magnitude and total number of earthquakes in a region and in a definite time interval. The GBR law is $[3,21]$ :

$$
\log (N)=a-b * M
$$

where $N$ is the number of the events, $M$ is the magnitude of the events, and " $a$ " and " $b$ ", so called b-value, are two empirical constants. The constant " $a$ " depends on the seismicity rate which varies greatly from region to region, whereas the constant " $b$ " is related to the properties of the material.
In order to obtain the b-value, the "maximumlikelihood estimation" approach is used. It is described with the following relationship [22]:

$$
b=\log (e) /\left(M_{a v}-M_{\min }\right)
$$

where $M_{a v}$ is the average of the observed magnitudes, and $M_{\min }$ is the minimum or the considered threshold magnitude. The standard error of the maximum likelihood estimation of $b$ is approximately $b / \sqrt{ } N$, for large $N$ (the number of earthquakes with $M \geq M \min$ ). The b-value follows the $\chi^{2}$ statistical distribution [22].

The GBR law shows that the probability of occurrence of a low magnitude event is higher in comparison with events characterized by very high magnitude.

Fig. 2 plots the cumulative number of earthquakes in the observation area versus magnitude. The plot refers to 240 events characterized by a magnitude greater or equal to 3.5 , of the Richter scale, in the geographic area of Jordan, Syria and south of Turkey [23]. The scale is logarithmic. It shows a decreasing of the curve when the magnitude of the earthquakes is important and equal to maximum value recording during the observation time interval.

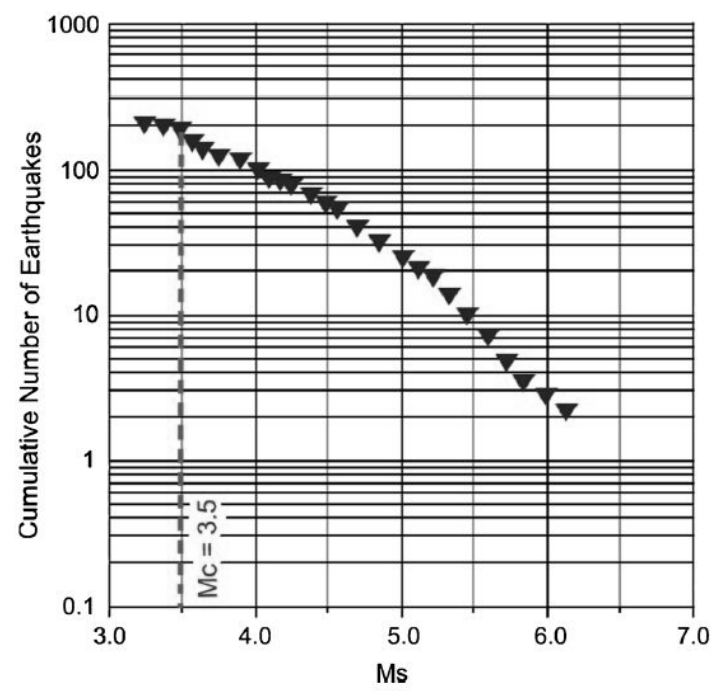

Fig. 2 - Cumulative distribution: the dashed line indicates the breakpoint, defining on the abscise the magnitude of completeness [23]

\subsection{GBR LAW FOR AE SIGNAL ANALYSIS}

There are more analogies between the AEs and the waves generated during the earthquakes. Similarly to Fig.2, the occurrences of AEs generated in the material under investigation increase in the low amplitude range of $\mathrm{A}_{\mathrm{dm}}$ and decrease in the high amplitude one. 
Moreover, the $\mathrm{AE}$ signals acquired during the tests are oscillating damped waves characterized by amplitude decreasing in the time. These waves are very similar to the $\mathrm{P}$ waves generated during an earthquake, and cause compression stress and dilatation in the crossed material. Moreover, both waves have a high speed that depends on the physical and morphological characteristics of the material in which they propagate.

On the basis of the analogies with the earthquake signal, in this paper is proposed to analyse the events generating the AE signals by the GBR law (1), modified as:

$$
\log (N)=a-b * A_{d m}
$$

where $N$ is the number of the hits of the AE signals higher than the noise threshold in the observation time interval, $A_{d m}$ is the maximum amplitude of $\mathrm{AE}$ event.

In the seismic, the b-value tends to decrease in the case earthquakes have high magnitude and it is equal to 1 [24]. In the proposed analysis, it is considered that the most important cracks, that occurs in the material investigated during the test, will generate AEs characterized by amplitude greater or equal to maximum value recorded during the test. For this AEs the b-value is set equal to 1 .

The value of $a$ is:

$$
a=\log (N)+b \cdot A_{d m}
$$

The trend of b-value is evaluated by:

$$
b=\frac{a-\log (N)}{A_{d m}}
$$

\section{PRELIMINARY EXPERIMENTAL RESULTS}

The aim of the test is to highlight correspondence between the critical AE signals and the cracks in the material.

All the data reported in this work refer to experiments carried out on cubic specimens in the Structures Laboratory of the University of Calabria. The specimens were designed to a concrete material with Rck $25 \mathrm{~N} / \mathrm{mm}^{2}$ to represent the behavior of a material used in the common structures [3].

The AE signals generated in the concrete specimen [7] are in the frequency range $[15,64] \mathrm{kHz}$. The acquisition system has 4 input channels, sampling frequency $5 \mathrm{MHz}, 12 \mathrm{bit}$ ADC resolution. The transducers connected to the input channel of the L-FAT are the R15 $\alpha$ [25].
The preliminary experimental tests were executed by a hydraulic press with a closed loop governing system with $3000 \mathrm{kN}$ and accuracy class $0.5 \%$ implementing a controlled uniaxial compression of the specimen with a constant displacement rate of $0.1 \mathrm{~mm} /$ minute until failure. The specimen under test is concrete cubic with no steel reinforcement, with size $15 \times 15 \times 15 \mathrm{~cm}^{3}$ (Fig. 3). According to EN12390-3 the samples are cured 28 days at the temperature $20 \pm 2{ }^{\circ} \mathrm{C}$, and relative humidity equal to $95 \%$.

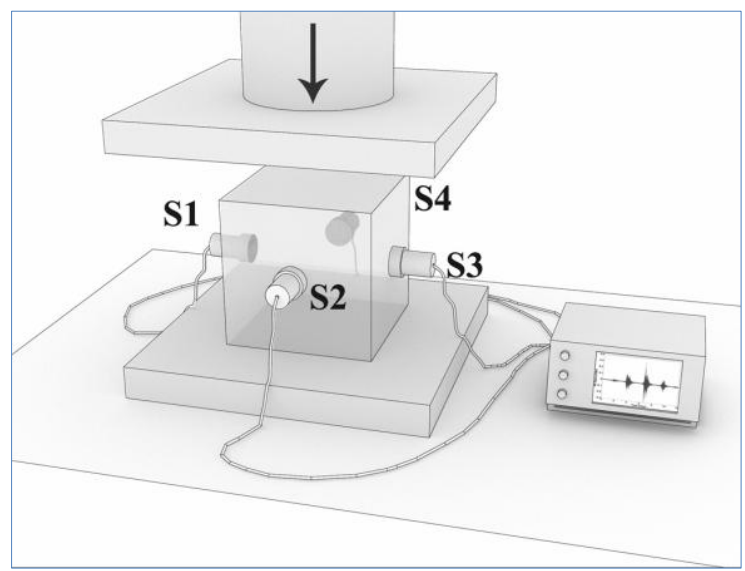

Fig. 3 -Concrete specimen used during the test and position of the piezoelectric transducers

In order to identify the critical events that occurs in the specimen during the test, the value of "a" is calculated experimentally by (4). The amplitude $A_{d m}$ has been imposed greater than $10 \mathrm{~V}$, because it is the minimum value that identifies the important cracks in the material, and b-value is assumed equal to 1 .

Fig. 4 shows the trend of $\log (\mathrm{N})$, experimentally evaluated and the "a" value estimated by (4) versus the number of critical events. It is experimentally evaluated $a=14.5$.

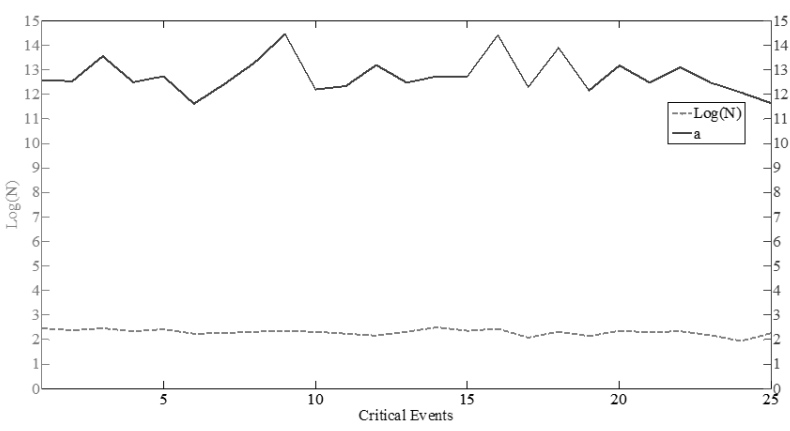

Fig. 4 - Experimental trend of $\log (N)$ and $a$ for critical AE

The b-value is calculated for each single acoustic emission during the compression test by the (5). Tab. 1 shows the number of events characterized by amplitude higher of the threshold noise versus mean 
b-value. The amplitude occurrences are divided in six ranges. The higher number of events occurs in the first range and then in the second one, that are associated at lower amplitude values. The mean bvalue decreases as the amplitude increase.

Table 1. Occurrences of cracks characterized by $\boldsymbol{A}_{d m}$ higher of the threshold noise versus $b$-value and.

\begin{tabular}{|c|c|c|}
\hline$A_{d m}[\mathrm{~V}]$ & Occurrence & mean b value \\
\hline $1-2$ & 410 & 7.4 \\
\hline $2-4$ & 331 & 4.2 \\
\hline $4-6$ & 102 & 2.8 \\
\hline $6-8$ & 42 & 1.9 \\
\hline $8-10$ & 21 & 1.5 \\
\hline $10-12$ & 40 & 1.1 \\
\hline
\end{tabular}

When the value is equal to unity the AE identifies important crack in the specimen under compression. Different types of cracks generate different $\mathrm{AE}$ signals with different frequency ranges and amplitudes. The b-value change and it is related to weak or strong events. The micro-cracks generate a large number of weak acoustic emissions, which leads to a relatively high b-value while macrocracking leads to a low b-values near to the unit, because they generated a large cracks and are characterized by amplitudes in range of $10-12 \mathrm{~V}$. The application of the technique permits to identify the fracture behavior in concrete.

Fig. $5 \mathrm{~b}$ shows the trend versus the time of the superposition of the cumulative number of critical AE signals characterized by the b-value equal to 1 . The frequency of critical AE signals, characterized by $b$ value in the neighbour equal to 1 , is increasing. The trend of this curve increases with the increasing of the stress in the specimen during the test (Fig. 5a). The specimen is characterized by non-uniform surface, and this causes, in the face subjected to compression, AE signals characterized by b-value near to 1 . These irregularities cause concentrated loads that create important cracks that propagate in a short time until settling of the test. In order to obtained an analysis that considers a state of art of the concrete similar to that used in construction, the $\mathrm{AE}$ signals detected during the first $600 \mathrm{~s}$, corresponding to the compression of $1 \mathrm{~mm}$ of specimen are discarded because generated by the irregular surface of the specimen. After this time interval, the settling of the specimen is finished, because there is a load stabilization and the increasing slope of the stress.

After that, it is obtained, Fig. $5 \mathrm{c}$, the AE event frequency estimated with window of $60 \mathrm{~s}$, sliding and step equal to $1 \mathrm{~s}$. This highlights that for the $\mathrm{AE}$ event frequency equal to 0.05 events/s the stress is equal to the $67.4 \%$ respect to the characteristic cubic compressive strength of concrete (Rck) equal to $25 \mathrm{MPa}$. On the basis of this analysis, the test is repeat for another 2 specimens. Also in these cases, in the observation window of $60 \mathrm{~s}$, the 3 critical $\mathrm{AE}$ signals indicates the increasing of the rate of the event, and then that the compressive strength is in the proximity of the $70 \%$ with respect to the maximum.
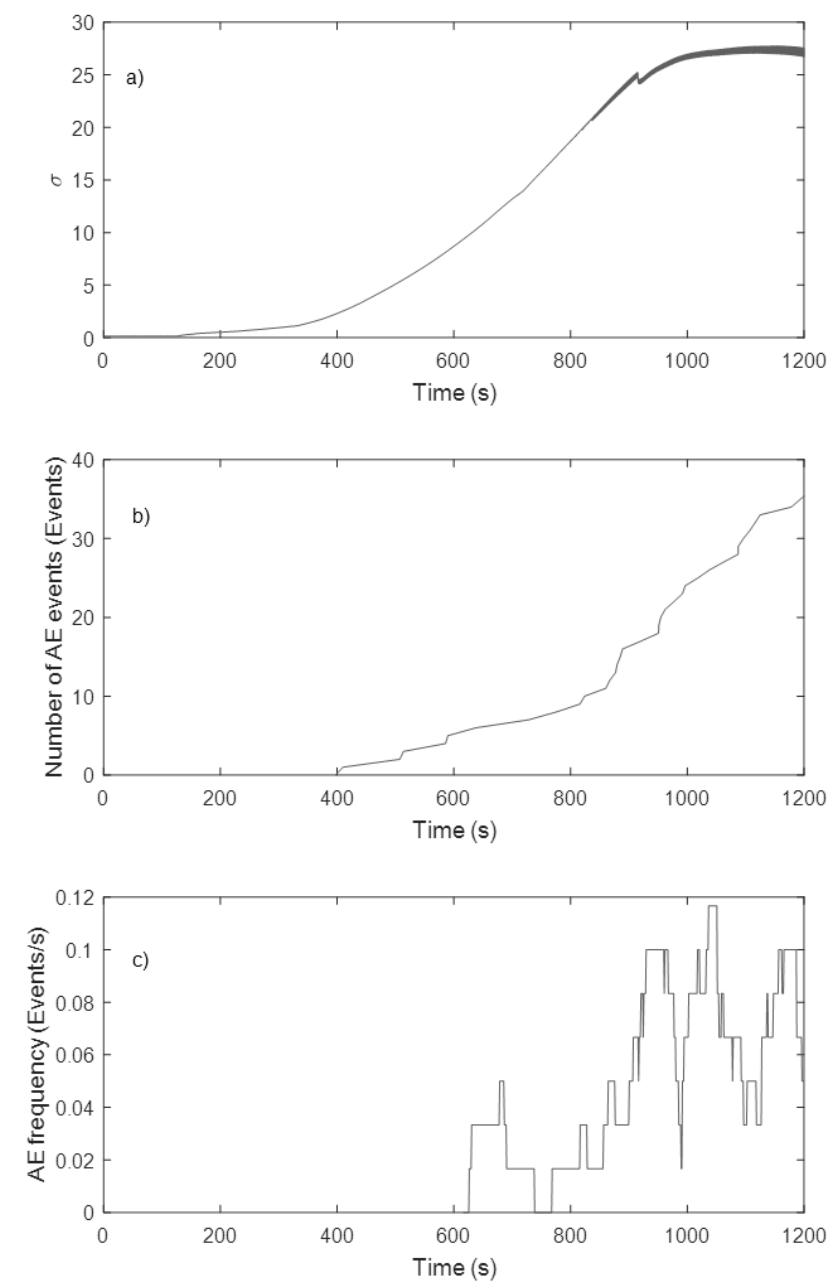

Fig. 5 - Trend of the a) stress versus time, b) cumulative number of critical AE signals detected (with $b=1$ ) versus time for specimen, and c) AE event frequency estimated with the sliding window with size equal to $60 \mathrm{~s}$ and step equal to $1 \mathrm{~s}$

\section{CONCLUSIONS}

In the paper a non-invasive technique for the damage evaluation of concrete structure is proposed. The technique is based on the analysis of Acoustic Emission (AE) signal and adaptation of the Gutenberg-Richter (GBR) law.

The adaptation of the GBR law permits to identify the critical damages. By evaluating the frequency of the critical $\mathrm{AE}$ signals the relationship between the AE signals and the specimen stress is highlighted. 
Preliminary experimental results confirm the analysis and shown that for the AE event frequency equal to 0.05 events/s the stress is close to the $70 \%$ of the maximum stress.

\section{REFERENCES}

[1] F. Lamonaca, A. Carrozzini, "Non destructive monitoring of civil engineering structures by using time frequency representation," in proceedings of the IEEE International Workshop on Intelligent Data Acquisition and Advanced Computing Systems: Technology and Applications, Rende (Cosenza), Italy, 21-23 September 2009.

[2] F. Lamonaca, D. L. Carnì, A. Carrozzini, D. Grimaldi, and R. S. Olivito, "Multitriggering and signal extraction for acoustic emissions monitoring," in Proceedings of the IEEE International Workshop on Metrology for Aerospace, MetroAeroSpace'2014, 2014, pp. 383-387

[3] D. L. Carnì, C. Scuro, F. Lamonaca, R. S. Olivito, D. Grimaldi., "Damage analysis of concrete structures by means of b-value technique," Composites Part B, Vol. 115, pp. 79-86, 2017.

[4] G. Lacidogna, F. Accornero, M. Corrado, and A. Carpinteri, "Crushing and fracture energies in concrete specimens monitored by Acoustic Emission," in Proceedings of the VIII International Conference on Fracture Mechanics of Concrete and Concrete Structures FraMCoS-8, 2013, pp. 1726-1736.

[5] M. V. M. S. Rao and K. J. Prasanna Lakshmi, "Analysis of b-value and improved b-value of acoustic emissions accompanying rock fracture," Current Science, Vol. 89, No. 9, pp. 1577-1582, 2005.

[6] C. Grosse, H. Reinhardt, and T. Dahm, "Localization and classification of fracture types in concrete with quantitative acoustic emission measurement techniques," Nondestructive Testing and Evaluation, Vol. 30, No. 4, pp. 223-230, Aug. 1997.

[7] M. E. Zitto, R. Piotrkowski, A. Gallego, F. Sagasta, and A. Benavent-Climent, "Damage assessed by wavelet scale bands and b-value in dynamical tests of a reinforced concrete slab monitored with acoustic emission," Mech. Syst. Signal Process., Vol. 60, pp. 75-89, 2015.

[8] B. Goszczyńska, G. Świt, and W. Trąmpczyński, "Application of the IADP acoustic emission method to automatic control of traffic on reinforced concrete bridges to ensure their safe operation," Arch. Civ. Mech. Eng., Vol. 16, No. 4, pp. 867-875, 2016.
[9] S. G. Shah and J. M. Chandra Kishen, "Use of acoustic emissions in flexural fatigue crack growth studies on concrete," Engineering Fracture Mechanics, Vol. 87, pp. 36-47, 2012.

[10] G. Siracusano, F. Lamonaca, R. Tomasello, F. Garescì, A. L. Corte, D. L. Carnì, M. Carpentieri, D. Grimaldi, and G. Finocchio, "A framework for the damage evaluation of acoustic emission signals through HilbertHuang transform," Mech. Syst. Signal Process., Vol. 75, pp. 109-122, 2015.

[11] C. U. Grosse and M. Ohtsu, Acoustic Emission Testing. Basic for Research Applications in Civil Engineering, Leipzig, Ger. Springer, 2008.

[12] S. Huguet, N. Godin, R. Gaertner, L. Salmon, and D. Villard, "Use of acoustic emission to identify damage modes in glass fibre reinforced polyester," Compos. Sci. Technol., Vol. 62, No. 10, pp. 1433-1444, 2002.

[13] R. Vidya Sagar and B. K. Raghu Prasad, "Damage limit states of reinforced concrete beams subjected to incremental cyclic loading using relaxation ratio analysis of $\mathrm{AE}$ parameters," Constr. Build. Mater., Vol. 35, pp. 139-148, 2012.

[14] O. Yapar, P. K. Basu, P. Volgyesi, and A. Ledeczi, "Structural health monitoring of bridges with piezoelectric AE sensors," Eng. Fail. Anal., Vol. 56, pp. 150-169, 2015.

[15] S. Kashif Ur Rehman, Z. Ibrahim, S. A. Memon, and M. Jameel, "Nondestructive test methods for concrete bridges: A review," Constr. Build. Mater., Vol. 107, pp. 58-86, 2016.

[16] F. Lamonaca, A. Carrozzini, D. Grimaldi, and R. S. Olivito, "Acoustic emission monitoring of damage concrete structures by multi-triggered acquisition system," in Proceedings of IEEE International Instrumentation and Measurement Technology Conference (I2MTC), 13-16 May, 2012,, pp. 1630-1634.

[17] O. Sano, M. Terada, and S. Ehara, "A study on the time-dependent microfracturing and strength of Oshima granite," Tectonophysics, Vol. 84, No. 2-4, pp. 343-362, April 1982.

[18] E. Verstrynge, K. Van Balen, M. Wevers, B. Ghiassi, and D. V. Oliveira, "Detection and localization of debonding damage in composite-masonry strengthening systems with the acoustic emission technique," in Proceedings of the International Conference on Emerging Technologies in Nondestructive Testing, ETNDT, Brussels, Belgium, 2016, pp. 511-517.

[19] B. Ghiassi, E. Verstrynge, P. B. Lourenço, and D. V. Oliveira, "Application of acoustic 
emission technique for bond characterization in FRP-masonry systems," Key Engineering Materials, 2015, vol. 624, pp. 534-541.

[20] E.J. Gumbel, Statistics of Extremes, Columbia University Press, New York, 1958.

[21] B. Gutenberg and C. F. Richter, "Magnitude and energy of earthquakes," Nature, Vol. 176, No. 4486, p. 795, 1955.

[22] A., Udias, and J., Mezcua, Fundamentos de Geofisica, Alianza Universidad Textos, 1997, $476 \mathrm{p}$.

[23] J. Asfahani, R. Darawcheh, "Seismicity assessment in and around Syria based on instrumental data: application of Gumbel distributions and Gutenberg-Richter relationship," Arabian Journal of Geosciences, Vol. 10, Issue 4, 2017.

[24] R. Vidya Sagar, "A parallel between earthquake sequences and acoustic emissions released during fracture process in reinforced concrete structures under flexural loading," Case Study in Construction Materials, Vol. 114, pp. 772-793, 2016.

[25] R. S. Olivito, C. Scuro, R. Codispoti, "Static analysis of masonry kilns built with fictile tubules bricks," in AIP Conference Proceedings, 2016.

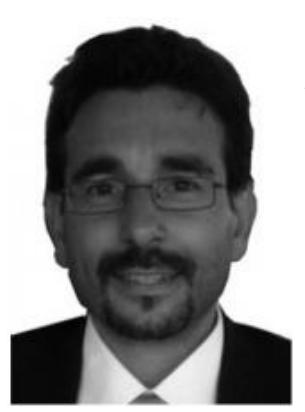

Domenico Luca Carnì, received the master's degree in computer engineering and the Ph.D. degree in systems and computer engineering from the University of Calabria, Rende, Italy, in 2003 and 2006, respectively.

In 2006, he joined the Department of Informatics, Mo-

deling, Electronics and Systems, University of Calabria, as an Assistant Professor of Electric and Electronic Measurements. His current research interests include the measurement on telecommunication systems, characterization of digital to analog and analog to digital converters, digital signal processing for monitoring and testing, virtual instrumentation, and distributed measurement systems.

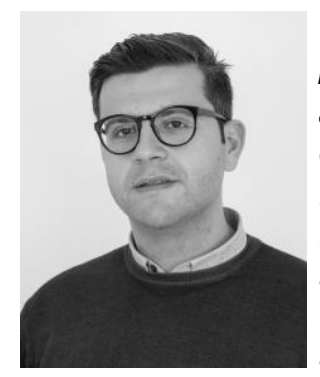

Carmelo Scuro, received the master's degree in Engineering and Architecture and the Ph.D. degree in Structural Mechanics from the University of Calabria, Rende, Italy, in 2012 and 2017, respectively.

His current research interests include the analysis of the fracture in the brittle materials through the use of Acoustic Emissions and structural monitoring systems, mechanical and physical characterization of traditional masonry techniques and experimentation on FRCM.

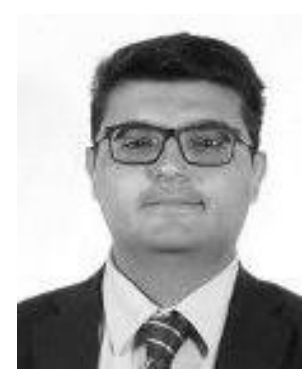

Francesco Lamonaca, received the M.S. degree in computer science engineering and the Ph.D. degree in computer and system science from the University of Calabria, Rende, Italy, in 2005 and 2010, respectively, and the doctorate equivalent degree in science and engineering science from the Université Libre de Bruxelles, Brussels, Belgium, in 2010 and 2011, respectively. He is currently an Associate Professor of Electronic Measurements with the University of Sannio, Benevento, Italy. He has authored and coauthored more than 150 papers published in international journals and conference proceedings. His current researches interests include measurement for medical use, characterization of human tissue by thermal analysis, digital signal and image processing for health parameters monitoring, noninvasive monitoring and testing, synchronization of networking measurement instruments and sensors, and distributed measurement systems. Dr. Lamonaca is a Senior member of the Institute of Electrical and Electronic Engineers (IEEE), and the Italian Group of Electrical and Electronic Measurements.

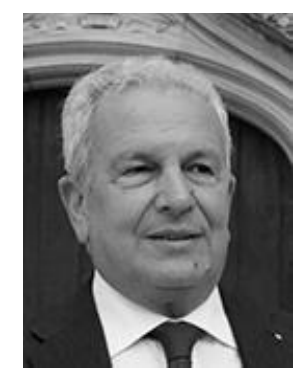

Renato S. Olivito, is a Full Professor of Structural Mechanics with the Department of Civil Engineering University of Calabria, Rende, Italy, where he has remained in a variety of research and management positions.

He was responsible for the research unit in the frame of the National Projects PRIN and RELUIS.

He was responsible for the PARCO Project supported by Calabria Region. He has authored or co-authored more than 150 papers published in international journals and conference proceedings. His current research interests include the ecosustainable composite materials as a strengthening of modern and historical masonry constructions, experimentation on FRCM and monitoring of structural through the use of Acoustic Emissions.

He is member of AIAS. 


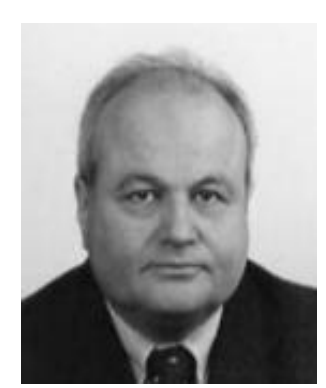

Domenico Grimaldi, is a Full Professor of Electronic Measurement with the Department of Computer Sciences, Modeling, Electronics, and System Science, University of Calabria, Rende, Italy, where he has remained in a variety of research and management positions.

$\mathrm{He}$ is responsible for the Laboratory for processing the measurement information. He was responsible for the research unit in the frame of the National Projects PRIN, FIRB, and international projects INTERLINK supported by the Italian Ministry for University and Research. He was responsible for the Tempus Project and Leonardo da Vinci Project supported by the European Union, and the PARCO Project supported by Calabria Region.
$\mathrm{He}$ is the delegate of the Rector of the University of Calabria for the safety. He has authored or coauthored more than 250 papers published in international journals and conference proceedings. His current research interests include the characterization of measurement transducers, digital signal processing for monitoring and testing, distributed measurements and synchronization, and measurement for medical application. The results of his research have led to a number of awards.

Prof. Grimaldi is a member of the Italian Group of Electrical and Electronic Measurements. $\mathrm{He}$ is member of the Editorial board of International Journal of Computing, Associate Editor of IEEE Transactions on Instrumentation and Measurement, and of Metrology and Measurement Systems. 